\title{
SÍNDROME DE APNEA HIPOAPNEA OBSTRUCTIVA DEL SUEÑO EN PACIENTE CON ACCIDENTE CEREBROVASCULAR ISQUÉMICO
}

Eduardo Palacios M D *, M arco A urelio R eyes M D**, Natalia Vega M D***, Diana M ejía M D****

\section{Resumen}

El síndrome de apnea hipoapnea obstructiva del sueño es considerado factor de riesgo para accidente cerebrovascular, que es a nivel mundial la segunda causa de muerte y primera de discapacidad. Objetivo: describir la prevalencia de sospecha de SAHOS por escala Epwhort y aumento de circunferencia cervical en pacientes con ACV isquémico en los hospitales de San José e Infantil Universitario de San José, de Bogotá DC, de octubre 2013 a septiembre 30 de 2014. Metodología: estudio descriptivo de corte transversal con población elegida con diagnóstico de $\mathrm{ACV}$ isquémico. Resultados: 125 pacientes con edad promedio de 69 años (DE 15.0) y relación hombre/mujer 1:1. Antecedente de SAHOS 1.6\% (n: 2). Por Epworth 57\% (n: 63/109) tenian sospecha de SAHOS, hombres 60,3\%(n: 35). La mediana para Epworth fue 10 (RIQ 7-12). Circunferencia cervical $>43 \mathrm{~cm}$ en hombres $8,6 \%$ (n:5) y en mujeres $>40 \mathrm{~cm}$ en $28 \%$ (n:14). El 30\% (19/62) de ACV tenía sospecha de SAHOS por Epworth y circunferencia cervical. Los hombres con Epworth (8-15), tenían circunferencia cervical aumentada 14\% (5/35) y las mujeres 51,9\% (14/27). La mortalidad intrahospitalaria fue 9,7\% (n:12). Conclusión: se encontró que más de la mitad tenía sospecha de SAHOS leve por Epworth. Hubo mayor prevalencia de obesidad cervical en mujeres y una tercera parte tenían sospecha de SAHOS por ambos criterios, lo cual confirma la importancia de explorar de rutina estos indicadores.

Palabras clave: accidente cerebrovascular agudo, síndrome de apnea hipoapnea obstructiva del sueño, factores de riesgo, circunferencia cervical, escala de Epworth.

Abreviaturas: ACV, accidente cerebrovascular; SAHOS, síndrome de apnea hipoapnea del sueño.

\section{OBSTRUCTIVE SLEEP APNEA HYPOAPNEA SYNDROME IN PATIENTS WITH ISCHEMIC CEREBROVASCULAR ACCIDENT}

\section{Abstract}

Obstructive sleep apnea hypoapnea syndrome is considered a risk factor for cerebrovascular accident which is the second cause of death and leading cause of disability worldwide. Objective: to describe suspicion prevalence of OSAHS estimated by the Epworth score and increase of neck circumference in patients with ischemic CVA at San José and Infantil Universitario de San José hospitals, Bogotá DC, from October 2013 and September 30

Fecha recibido: noviembre 6 de 2014 - Fecha aceptado: diciembre 10 de 2014

* Jefe del Servicio de Neurología, Hospital de San José. Miembro de la Sociedad de Cirugía de Bogotá, Colombia. Profesor Titular, Fundación Universitaria de Ciencias de la Salud. Bogotá DC, Colombia.

** Neurólogo Hospital de San José. Instructor Asistente, Fundación Universitaria de Ciencias de la Salud, Bogotá DC, Colombia.
*** Residente IV de Neurología, Fundación Universitaria de Ciencias de la Salud. Bogotá DC, Colombia.

**** Residente III de Neurología, Fundación Universitaria de Ciencias de la Salud. Bogotá DC, Colombia.

***** Grupo de Investigación INEUROPSI. Línea de investigación enfermedad cerebrovascular. 
2014. Methodology: cross sectional descriptive study including a population diagnosed with ischemic CVA. Results: 125 patients with mean age 69 years (SD 15.0) and male/female ratio of 1:1. History of OSAHS in 1.6\% (n: 2). By Epworth score, 57\% (n: 63/109) had suspicion of OSAHS, 60.3\% (n: 35) corresponded to men. The media for Epworth score was 10 (RIQ 7-12). Neck circumference, $>43 \mathrm{~cm}$ in men 8.6\% (n: 5) and women $>40 \mathrm{~cm}$ in $28 \%$ (n: 14). OSAHS was suspected in $30 \%$ of CVAs (19/62) by Epworth score and neck circumference. Men with Epworth score (8-15), had an increased neck circumference 14\% (5/35) and women $51.9 \%$ (14/27). In-hospital mortality was $9.7 \%$ (n: 12). Conclusion: we found that mild SOAHS estimated by Epworth score was suspected in more than half the patients. There was a higher prevalence of neck obesity in women and OSAHS was suspected in one third of them by both criteria, which confirms the importance of routinely exploring these indicators.

Key words: acute cerebrovascular accident, obstructive sleep apnea hypoapnea syndrome, risk factors, neck circumference, Epworth scale

\section{Introducción}

L a enfermedad cerebrovascular es una patología que puede llevar a un gran deterioro de la capacidad funcional, por lo cual es considerada como la primera causa de discapacidad en el mundo. R epresenta una al ta tasa de morbimortalidad presentándose hasta 700.000 casos por año en Estados U nidos. ${ }^{1}$ El metanálisis (stroke y apnea) determinó qué factores pueden influir en su frecuencia. ${ }^{2}$ El riesgo de recurrencia es de $10 \%$ en una semana, entre $2 \%$ y $4 \%$ al mes. $^{3}$

EI SA HOS es causado por obstrucción del flujo de aire secundario al colapso de la vía aérea superior, aunque el esfuerzo respiratorio está todavía presente. Su definición se ha planteado como más de cinco episodios de apnea medidos por polisomnograma. El índice de apnea e hipopnea (IA H) se ha utilizado para clasificar el SA HOS en leve ( 5 a 15 eventos por hora), moderado (15 a 30) y severo (mayor de 30).3,4

La apnea del sueño es un factor independiente que aumenta el riesgo de ACV o muerte hasta dos veces más que un paciente que no tenga SA HOS y dependiendo del grado de severidad en la escala de IAH también se asociará con mayor riesgo, hasta tres 0 cuatro veces, de desarrollar un ACV. ${ }^{5}$ Se ha documentado un aumento del riesgo de ACV o muerte en Ios pacientes con SAHOS a pesar de la utilización de múltiples terapias. ${ }^{6}$
Dentro de los estudios de factores de riesgo de enfermedad cerebrovascular (hipertensión arterial, diabetes, tabaquismo, obesidad) no se explora de rutina el SAHOS y si se estudian los factores de riesgo tradicionales. ${ }^{7}$ L os datos del Sleep Heart Health Study demostró que la edad, el índice de masa corporal (IMC), la circunferencia del cuello, ronquidos, la respiración y frecuencia de apneas se asociaron con SAHOS. ${ }^{8}$ El objetivo de la investigación es describir la prevalencia de sospecha de SA HOS por medio de dos indicadores: escala Epworth y aumento de la circunferencia cervical en pacientes que cursan con ACV isquémico, además de indagar por antecedente conocido de SA HOS y de esta manera poder orientar intervenciones diagnósticas o terapéuticas en nuestra población para reducir el riesgo de un segundo evento cerebrovascular.

\section{Métodos}

Se realizó un estudio de corte transversal, en pacientes mayores de 18 años, que consultaron por ACV isquémico al servicio de neurología de los hospitales de San J osé e I nfantil U niversitario de San J osé en el período comprendido entre el 1 de octubre 2013 y 30 de septiembre 2014. Los pacientes se diagnosticaron con un episodio de déficit neurológico agudo causado por isquemia cerebral, confirmado por neuroimagen cerebral. Se excluyeron embarazadas y ACV extenso con compromiso de tallo cerebral. La historia clíni- 
ca, el examen físico, y la interpretación inicial de la neuroimagen, fueron realizados por neurólogos; se documentaron antecedentes sobre factores de riesgo cardiovascular (HTA, diabetes mellitus tipo 2 y tabaquismo) entre otros.

La recolección de datos se realizó por medio de la historia clínica y un formato donde se incluyeron las variables de interés, las cuales se registraron en la base de datos. Se indagó por antecedente de SA HOS, realizando al paciente preguntas en relación con antecedente de ronquido y si había sido diagnosticado antes con SA HOS. Se les aplicó la escala E pworth, la cual evalúa la probabilidad de tener somnolencia en ocho situaciones cotidianas, esta escala tiene una sensibilidad de $60 \%$ y especificidad de $82 \%$. ${ }^{9}$ Se encuentra validada y con adaptación lingüística al idioma español. ${ }^{10}$

Se realizó medición estandarizada de la circunferencia cervical con cinta métrica, a nivel del cartilago cricoides, con puntos de corte para definir obesidad cervical en mujeres $(>40 \mathrm{~cm}$ ) y hombres $(>43$ $\mathrm{cm})$. A todos los pacientes en quienes se identificó aumento de la circunferencia cervical o puntaje en escala E pworth con sospecha de SA HOS $>8$ leve, $>16$ moderada y $>18$ severa, se les ordenó al egreso hospitalario polisomnograma y se le explicó a la familia y al paciente la importancia del examen y la necesidad de revisar los resultados en control por consulta externa. Se tabuló la información de los datos obtenidos en formato de recolección con sus variables correspondientes en el programa Microsoft Office Excel 2007. El protocolo fue aprobado por el comité de investigaciones de la Facultad de M edicina de la Fundación Universitaria de Ciencias de la Salud, el H ospital de San J osé y el Hospital Infantil U niversitario de San J osé.

\section{Análisis estadístic o}

Se hizo un análisis descriptivo utilizando el programa Stata 13.0. Las variables continuas se resumen con medidas de tendencia central y dispersión. L as categóricas se reportan con frecuencias obsolutas y relativas. Se reporta la preval encia de sospecha de SA HOS por escala E pworth y por circunferencia cervical, según la distribución por sexo y sospecha de SAHOS en pacientes que presentan a la vez puntaje en escala de Epworth $>8$ y obesidad cervical. Se utilizó el coeficiente de correlación de Spearman para evaluar la correlación lineal entre la circunferencia cervical y el puntaje en la escala de E pworth.

\section{Resultados}

Ingresaron 125 pacientes con ACV isquémico. En la Tabla 1 se muestran las características de la población en estudio; edad promedio 69 años (DE 15.0), relación hombre/mujer 1:1, antecedente de hipertensión arterial $70,2 \%$, dislipidemia $13,8 \%$ y sobrepeso $40,3 \%$. El $20 \%$ tenía antecedente personal de enfermedad cerebrovascular, de los cuales $48,1 \%$ tenía un Rankin mayor a 4 . El 1,6\% (n:2) de los pacientes tenia antecedente de SAHOS al ingreso del estudio.

Por la escala de E pworth el 57\% (n: 63/109) tenía sospecha de SA HOS; en hombres: $60,3 \%$ ( $n: 35$ ) y mujeres $54,9 \%$ (n:28), todos con puntuaciones entre 8 y 15 (SA HOS leve). La mediana en el puntaje de la escala Epworth fue de 10 (RIQ 7-12) y para circunferencia cervical fue de $38 \mathrm{~cm}$ (RIQ 36-40). La circunferencia cervical en hombres $>43 \mathrm{~cm}$ fue de $8,6 \%$ (n:5) y mujeres $>40 \mathrm{~cm}$ de $28 \%$ (n:14). El 30\% (19/62) de los pacientes con ACV tenían tanto sospecha de SAHOS por escala Epworth como por aumento de circunferencia cervical. L os hombres con E pworth $>9$ y con circunferencia cervical aumentada fueron $14 \%$ (n:5/35) y mujeres 51,9\% (n:14/27) Tabla 2.

En el análisis de la relación entre la circunferencia cervical y puntaje de E pwoth se encontró una correlación lineal positiva moderada ( $\rho=0.53 ; p<0.0001$ ). Durante el desarrollo del estudio se encontró una mortalidad intrahospitalaria de 9,7\% (n:12).

\section{Disc usión}

EI SA HOS en un síndrome considerado factor de riesgo independente para enfermedad cerebrovascular, siendo frecuente y bien documentado en la literatura. En 1991 J ohns y col. desarrollaron la escala E pworth como indicador de somnolencia diurna y se estableció 


\begin{tabular}{|c|c|c|c|c|c|c|}
\hline \multirow[b]{2}{*}{ Edad, años, promedio (DE) } & \multicolumn{2}{|c|}{ Hombres (n: 63 ) } & \multicolumn{2}{|c|}{ Mujeres (n:62 ) } & \multicolumn{2}{|c|}{ Total ( $\mathrm{n}: 125)$} \\
\hline & 67.7 & $(13,6)$ & 70.6 & $(16,2)$ & 69.1 & $(15,0)$ \\
\hline HTA, n (\%) & 42 & $(67,7)$ & 45 & $(72,5)$ & 87 & $(70,2)$ \\
\hline DM, n (\%) & 4 & $(6,5)$ & 8 & $(13,3)$ & 12 & $(9,9)$ \\
\hline Alteración tiroidea, n (\%) & 6 & $(9,52)$ & 14 & $(22,58)$ & 20 & $(16,0)$ \\
\hline hipotiroidismo & 2 & $(3,2)$ & 8 & $(12,9)$ & 10 & $(8,0)$ \\
\hline hipertiroidismo & 0 & & 1 & $(1,6)$ & 1 & $(0,8)$ \\
\hline Dislipidemia & 7 & $(11,3)$ & 10 & $(16,4)$ & 17 & $(13,8)$ \\
\hline IMC, promedio (DE) & 26.2 & $(3,11)$ & 24.7 & $(4,16)$ & 25.5 & $(3,73)$ \\
\hline bajo peso & - & - & 3 & $(7,7)$ & 3 & $(3,9)$ \\
\hline normal & 16 & $(42,1)$ & 19 & $(48,7)$ & 35 & $(45,5)$ \\
\hline sobrepeso & 18 & $(47,4)$ & 13 & $(33,3)$ & 31 & $(40,3)$ \\
\hline obesidad GI & 4 & $(10,5)$ & 3 & $(7,7)$ & 7 & $(9,1)$ \\
\hline obesidad GII & & & 1 & $(2,6)$ & 1 & $(1,3)$ \\
\hline Fuma, n(\%) & 28 & $(44,4)$ & 12 & $(19,35)$ & 40 & $(32,0)$ \\
\hline Alcohol, n(\%) & 18 & $(28,57)$ & 2 & $(3,23)$ & 20 & $(16,0)$ \\
\hline Enf. carotídea, n(\%) & - & - & - & - & - & - \\
\hline Fibrilación auricular, n(\%) & 5 & $(7,94)$ & 4 & $(6,45)$ & 9 & $(7,2)$ \\
\hline IAM, n(\%) & 6 & $(90,4)$ & 3 & $(4,84)$ & 9 & $(7,2)$ \\
\hline Falla cardíaca, n(\%) & 3 & $(4,76)$ & 5 & $(8,06)$ & 8 & $(6,4)$ \\
\hline Antecedente de ACV, n(\%) & 15 & $(23,81)$ & 10 & $(16, I)$ & 25 & $(20,0)$ \\
\hline \multicolumn{7}{|l|}{ Rankin $\geq 4$ 1, n(\%) } \\
\hline 1 & & & & & I & $(4,0)$ \\
\hline 2 & & & & & 6 & $(24,0)$ \\
\hline 3 & & & & & 6 & $(24,0)$ \\
\hline 4 & & & & & 12 & $(48, I)$ \\
\hline Antecedente conocido SAHOS, n(\%) & 0 & & 2 & $(3,23)$ & 2 & $(1,6)$ \\
\hline $\mathrm{ACV}$ isquémico & 61 & $(96,8)$ & 62 & $(100,0)$ & 123 & $(98,4)$ \\
\hline $\begin{array}{l}\text { ACV isquémico con transformación } \\
\text { hemorrágica }\end{array}$ & 2 & $(3,17)$ & 0 & & 2 & $(1,6)$ \\
\hline Traslado UCl, n(\%) & 16 & $(25,4)$ & 19 & $(3 \mathrm{I}, \mathrm{I})$ & 35 & $(28,23)$ \\
\hline Muerte intrahospitalaria & & & & & 12 & $(9,7)$ \\
\hline
\end{tabular}


Tabla 2. Indicadores de sospecha de SAHOS en la población con ACV isquémico $n=108$

\begin{tabular}{|l|c|c|c|}
\hline \multicolumn{2}{|c|}{$\begin{array}{c}\text { Hombres } \\
\text { n:50 }\end{array}$} & $\begin{array}{c}\text { Mujeres } \\
n: 58\end{array}$ & Total \\
\hline$>8$ leve sospecha SAHOS & $35(60,3)$ & $28(54,9)$ & \\
\hline$>16$ moderada sospecha SAHOS & & & 10 \\
\hline$>18$ severa sospecha de SAHOS & & & \\
\hline Mediana de escala de Epworth & $10(8-15)$ & $10(8-15)$ & \\
\hline Hombres & & & $58(100,0)$ \\
\hline Mujeres & $5(14,2)$ & $14(51,9)$ & $50(100,0)$ \\
\hline Circunferencia cervical/ escala Epwhort & & & \\
\hline \multicolumn{1}{|c|}{ hombres $>43.1 \mathrm{~cm} / 8$} & & & \\
\hline mujeres $>40 \mathrm{~cm} / 8$ & & & \\
\hline
\end{tabular}

una relación con sospecha de SAHOS. Es razonable presumir que el SAHOS es un factor de riesgo cerebrovascular dada su fisiopatología, desencadena estrés oxidativo acumulativo dentro de la vasculatura y afecta en gran medida la función endotelial, inflamación vascular y la aterosclerosis $s^{11}$, por lo cual sería prevenible y aunque esta hipótesis no es del todo aceptada, consideramos que es necesario establecer una sospecha clínica adecuada para hacer una elección óptima de pacientes que irán a polisomnograma y asi poder hacer el diagnóstico y mejorar los desenlaces en nuestros pacientes, ya que han sido reportados datos suficientes para relacionar la recurrencia de ACV y resultados desfavorables en estos pacientes. ${ }^{12,13}$

En nuestro estudio encontramos que el $57 \%$ de los pacientes tenian sospecha de SAHOS por escala E pworth, que es similar a lo reportado en la literatura. $M$ edeiros y coll $^{14}$ analizan varios cuestionarios entre ellos la escala E pworth y sospecha de SA HOS, estudio realizado en 89 pacientes, donde encontraron que en el $52 \%$ había sospecha clínica por esta escala. Hay que reconocer que ambos estudios se realizaron durante la estancia hospitalaria del evento agudo y no se realizó confirmación con la prueba de oro para SA HOS que es el polisomnograma.
En relación con la circunferencia cervical, los resultados de nuestro estudio se comparan con lo reportado por M edeiros y col. donde las mujeres tienen mayor prevalencia de obesidad cervical ${ }^{14}$; encontramos que este es un marcador de obesidad central y se ha asociado con apnea del sueño como riesgo cardiovascular.

Las limitaciones del presente estudio para el tamizaje de SAHOS con la prueba de oro el polisomnograma no se realizó durante la hospitalización debido a la condición clínica y neurológica que presentaban los pacientes con A C V. En el seguimiento en consulta externa ningún paciente regresó con resultados de polisomnograma informando multiples causas, entre ellas no autorización por parte de empresa prestadora de servicio de salud, vencimiento de la orden médica, pérdida de la orden y olvido del paciente.

En la práctica clínica la manera rápida y reproducible de sospechar SA HOS es con la aplicación de la escala de Epworth y la medición de la circunferencia cervical, para tomar medidas terapéuticas inmediatas y ampliar estudios requeridos como el polisomnograma. 


\section{Conclusiones}

Se encontró que más de la mitad de los pacientes presentan sospecha de SA HOS leve por escala E pworth. En relación con la circunferencia cervical es mayor la prevalencia de obesidad cervical en mujeres que en hombres. ${ }^{15}$

U na tercera parte de los pacientes con ACV isquémico tienen sospecha de SA HOS por ambos criterios, lo cual confirma la importancia de la exploración de rutina de estos indicadores. A I tener la sospecha, diagnosticarlo y tratarlo podemos disminuir el riesgo de un evento cerebrovascular y por lo tanto restringir el riesgo de discapacidad por esta patología en nuestra población.

\section{Referencias}

1. Dyken ME, Im KB. Obstructive sleep apnea and stroke. Chest. 2009 Dec;136(6):1668-77

2. Johnson KG, Johnson DC. Frequency of sleep apnea in stroke and TIA patients: a meta-analysis. J Clin Sleep Med. 2010;6(2):131-7.

3. Balami JS, Chen RL, Grunwald IQ, Buchan AM. Neurological complications of acute ischaemic stroke. Lancet Neurol. 2011 Apr;10(4):357-71.

4. Norman D, Loredo JS. Obstructive Sleep Apnea in Older Adults. Clin Geriatr Med. 2008;24(1):151-65.
5. Ho ML, Brass SD. Obstructive sleep apnea. Neurol Int. 2011;3(3):e15.

6. Go AS, Mozaffarian D, Roger VL, Benjamin EJ, Berry JD, Borden WB, et al. Heart disease and stroke statistics--2013 update: a report from the American Heart Association. Circulation. 2013 Jan 1; 127(1):e6-e245.

7. Epstein LJ, Kristo D, Strollo PJ Jr, Friedman N, Malhotra A, Patil SP. Clinical Guideline for the evaluation, management and long-term care of obstructive sleep apnea in adults. J Clin Sleep Med. 2009 Jun 15; 5(3):263-76.

8. Yaggi HK, Concato J, Kernan WN, Lichtman JH, Brass LM, Mohsenin V. Obstructive sleep apnea as a risk factor for stroke and death. N Engl J Med. 2005;353(19):2034-41

9. Cohen-Zion M, Stepnowsky C, Marler, Shochat T, Kripke DF, Ancoli-Israel S Changes in cognitive function associated with sleep disordered breathing in older people. J Am Geriatr Soc. 2001; 49(12):1622-7.

10. Chica-Urzola HL, Escobar-Córdoba F, Eslava-Schmalbach J. [Validating the Epworth sleepiness scale]. Rev Salud Publica (Bogota). 2007 Oct-Dec;9(4):558-67.

11. Devulapally K, Pongonis R, Khayat R. OSA: the new cardiovascular disease: part II: Overview of cardiovascular diseases associated with obstructive sleep apnea. Heart Fail Rev. 2009;14(3):155-64.

12. Kuniyoshi FH, Pusalavidyasagar S, Singh P, Somers VK. Cardiovascular consequences of obstructive sleep apnoea. Indian J Med Res. 2010;131:196-205.

13. Birkbak J, Clark AJ, Rod NH. The Effect of Sleep Disordered Breathing on the Outcome of Stroke and Transient Ischemic Attack: A Systematic Review. J Clin Sleep Med.10(1):103-8.

14. Medeiros CA, Bruin VM, Castro-Silva Cd, Araújo SM, Chaves Junior M, Bruin PF.Neck circumference, bedside clinical feature related to mortality of acute ischemic stroke. Rev Assoc Med Bras. 2011 Sep-Oct; 57(5):559-64.

15. Whittle AT, Marshall I, Mortimore IL, Wraith PK, Sellar RJ, Douglas NJ. Neck soft tissue and fat distribution: comparison between normal men and women by magnetic resonanceimaging. Thorax. 1999 Apr; 54(4):323-8. 